\title{
Control of Stratification in Drying Particle Suspensions via Temperature Gradients
}

\author{
Yanfei Tang, ${ }^{\dagger}$ Gary S. Grest, ${ }^{\ddagger 0}$ and Shengfeng Cheng* ${ }^{* \dagger}{ }^{\dagger}$ \\ ${ }^{\dagger}$ Department of Physics, Center for Soft Matter and Biological Physics, and Macromolecules Innovation Institute, Virginia \\ Polytechnic Institute and State University, Blacksburg, Virginia 24061, United States \\ ${ }^{\ddagger}$ Sandia National Laboratories, Albuquerque, New Mexico 87185, United States
}

Supporting Information

ABSTRACT: A potential strategy for controlling stratification in a drying suspension of bidisperse particles is studied using molecular dynamics simulations. When the suspension is maintained at a constant temperature during fast drying, it can exhibit "small-on-top" stratification with an accumulation (depletion) of smaller (larger) particles in the top region of the drying film, consistent with the prediction of current theories based on diffusiophoresis. However, when only the region near the substrate is thermalized at a constant temperature, a negative temperature gradient develops in the
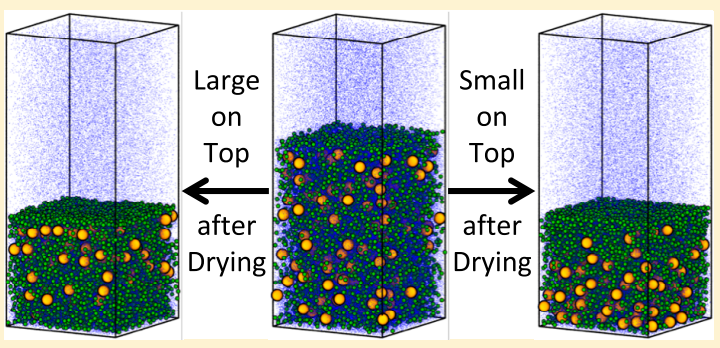
suspension because of evaporative cooling at the liquid-vapor interface. Since the associated thermophoresis is stronger for larger nanoparticles, a higher fraction of larger nanoparticles migrate to the top of the drying film at fast evaporation rates. As a result, stratification is converted to "large-on-top". Very strong small-on-top stratification can be produced with a positive thermal gradient in the drying suspension. Here, we explore a way to produce a positive thermal gradient by thermalizing the vapor at a temperature higher than that of the solvent. Possible experimental approaches to realize various thermal gradients in a suspension undergoing solvent evaporation and thus to produce different stratification states in the drying film are suggested.

\section{INTRODUCTION}

The drying of colloidal suspensions has been studied for several decades. ${ }^{1-17}$ Recently, drying-induced stratification phenomena in polydisperse colloidal mixtures have attracted great attention, ${ }^{6,7,18-33}$ as they point to a quick, facile, one-pot method of depositing layered multifunctional coating films on a surface. In a particle suspension undergoing drying, the vertical distribution of particles is controlled by the Péclet number, $\mathrm{Pe}=H v_{\mathrm{e}} / D$, where $H$ is the thickness of the suspension film, $v_{\mathrm{e}}$ is the receding speed of the liquid-vapor interface during evaporation, and $D$ is the diffusion coefficient of the particles. ${ }^{9,34}$ The Péclet number characterizes the competition between diffusion and evaporation-induced particle migration. When $\mathrm{Pe} \gg 1$, the particles build up near the interface and their final distribution in the dry film may develop gradients, whereas for $\mathrm{Pe} \ll 1$, the particles diffuse fast enough to mitigate evaporative effects and are expected to be uniformly distributed in the deposited film. ${ }^{9}$

In the case of a suspension of a bidisperse mixture of particles made from the same material but having different diameters, $d_{1}$ and $d_{s}$, the final distribution of particles is determined by two Péclet numbers, $\mathrm{Pe}_{1}$ and $\mathrm{Pe}_{\mathrm{s}}$, for the large and small particles, respectively. If the Stokes-Einstein relationship holds, then $\mathrm{Pe}_{1} / \mathrm{Pe}_{s}=d_{1} / d_{s}>1$. When $\mathrm{Pe}_{1}>1>$ $\mathrm{Pe}_{\mathrm{s}}$, Trueman et al. found the so-called "large-on-top" stratification, ${ }^{12,13}$ where the larger (smaller) particles are enriched (depleted) near the receding interface. Recently,
Fortini et al. discovered the counterintuitive "small-on-top" stratification in the regime of $\mathrm{Pe}_{1}>\mathrm{Pe}_{s} \gg 1$, i.e., when drying is extremely rapid. ${ }^{18,21}$ Since then, a number of experimental, $^{19,24,27,29,31,33}$ theoretical, ${ }^{20,25,26}$ and simulation ${ }^{22,23,28,30,32}$ studies have been reported on the stratification phenomena in drying suspensions of polydisperse particles and their physical mechanisms. The idea of diffusiophoresis being responsible for small-on-top stratification is widely supported. ${ }^{18,20,22,25,26,30}$ In this picture, when $\mathrm{Pe}_{s} \gg 1$, the smaller particles congregate near the receding interface during evaporation and their distribution develops a gradient that decays into the drying film. Further, when the volume fraction of the smaller particles, $\phi_{s}$, is above a certain threshold that depends on $\mathrm{Pe}_{s}$, this gradient generates a diffusiophoretic force that is strong enough to push the larger particles out of the interfacial region. Consequently, the larger particles are depleted near the interface, resulting in small-on-top stratification.

The key ingredient of the diffusiophoretic model is that the cross-interaction between the large and small particles has asymmetric effects on the phoretic drift of particles and drives the larger ones away from the interfacial region faster than the smaller ones. ${ }^{20,25}$ Therefore, the size asymmetry, quantified as $\alpha=d_{1} / d_{\text {s }}$, is a crucial parameter that controls the outcome of

Received: October 30, 2018

Revised: February 20, 2019

Published: February 26, 2019 
stratification, with larger $\alpha$ favoring small-on-top stratification. Martín-Fabiani et al. studied a system with the smaller particles coated with hydrophilic shells and explored the effect of changing the $\mathrm{pH}$ of the initial dispersion. ${ }^{19}$ In a dispersion with low $\mathrm{pH}, \alpha$ is large enough to lead to small-on-top stratification. When the $\mathrm{pH}$ is raised, $\alpha$ is reduced as the hydrophilic shells swell substantially and stratification is suppressed.

The approach of Martín-Fabiani et al. can be used for systems where the particle size can be tuned with external stimuli. ${ }^{19}$ However, other possible approaches of controlling stratification for systems with fixed particle sizes have rarely been explored. In a previous work, ${ }^{30}$ we used molecular dynamics (MD) modeling to study drying suspensions of a binary mixture of nanoparticles and found that for fast evaporation rates, the solvent can develop a negative temperature gradient toward the interface because of evaporative cooling effect. This temperature gradient induces thermophoresis, in which the larger particles are pushed more strongly into the interfacial region where the temperature is lower and the solvent density is higher. The competition between thermophoresis generated by evaporative cooling and diffusiophoresis can thus suppress small-on-top stratification at ultrafast drying rates or even turn the stratification into largeon-top. ${ }^{30}$ This discovery further indicates that thermophoresis, with a controlled thermal gradient other than the naturally occurring evaporative cooling, may be used to control stratification. In this paper, we employ MD modeling to test this idea in detail and demonstrate that stratification in a drying suspension can be controlled on demand with a temperature gradient imposed on the system, i.e., via controlled thermophoresis.

\section{METHODS}

We performed MD simulations on a suspension of a bidisperse mixture of nanoparticles. ${ }^{30}$ The solvent is modeled explicitly as beads of mass $m$ and interacting with each other via a Lennard-Jones (LJ) potential, $U_{\mathrm{LJ}}(r)=4 \epsilon\left[(\sigma / r)^{12}-(\sigma / r)^{6}-\left(\sigma / r_{\mathrm{c}}\right)^{12}+\left(\sigma / r_{\mathrm{c}}\right)^{6}\right]$, where $r$ is the center-to-center distance between beads, $\epsilon$ is an energy scale, $\sigma$ is a length scale, and the potential is truncated at $r_{\mathrm{c}}=3 \sigma$. The nanoparticles are modeled as spheres with a uniform distribution of $\mathrm{LJ}$ beads at a mass density $1.0 \mathrm{~m} / \sigma^{35,36}$ The large nanoparticles (LNPs) have diameter $d_{1}=20 \sigma$ and mass $m_{1}=4188.8 m$, and the small nanoparticles (SNPs) have diameter $d_{\mathrm{s}}=5 \sigma$ and mass $m_{\mathrm{s}}=65.4 \mathrm{~m}$. The size ratio is $\alpha=4$. The nanoparticle-nanoparticle interactions are given by an integrated form of the $\mathrm{LJ}$ potential for two spheres with a Hamaker constant, $A_{\mathrm{nn}}$, characterizing the interaction strength. ${ }^{35,36}$ In this study, $A_{\mathrm{nn}}=39.48 \epsilon$. To ensure that nanoparticles are well dispersed in the initial suspension, the nanoparticle-nanoparticle interactions are rendered purely repulsive by truncating them at $20.574 \sigma, 13.085 \sigma$, and $5.595 \sigma$ for the LNP-LNP, LNP-SNP, and SNP-SNP pairs, respectively. The nanoparticle-solvent interactions are described by a similar integrated form of the LJ potential with a Hamaker constant $A_{\mathrm{ns}}=100 \epsilon$ and a cutoff length $d / 2+4 \sigma$, where $d$ is the nanoparticle diameter. ${ }^{37}$ The nanoparticle-solvent interactions thus have attractive tails, which make the effective diameter of a nanoparticle larger than its nominal diameter. ${ }^{30}$ The size ratio is defined here on the basis of the nominal diameters of LNPs and SNPs. If their effective diameters are used, then the size ratio is about 3.4.

The entire system consists of $\sim 7 \times 10^{6} \mathrm{LJ}$ beads, $200 \mathrm{LNPs}$, and 6400 SNPs. The system is placed in a rectangular simulation cell of dimensions $L_{x} \times L_{y} \times L_{z}$, where $L_{x}=L_{y}=201 \sigma$ and $L_{z}=477 \sigma$. The liquid-vapor interface is in the $x-y$ plane, in which periodic boundary conditions are imposed. In the initial suspension, the thickness of the liquid film is about $304 \sigma$. The volume fractions of LNPs and SNPs in the initial dispersion are $\phi_{1}=0.068$ and $\phi_{\mathrm{s}}=0.034$, respectively. Along the $z$-axis, all particles are confined in the simulation cell by two walls at $z=0$ and $L_{z}$. The particle-wall interaction is given by an LJ-like 93 potential, $U_{\mathrm{W}}(h)=\epsilon_{\mathrm{W}}\left[(2 / 15)\left(D_{\mathrm{W}} / h\right)^{9}-\left(D_{\mathrm{W}} / h\right)^{3}-(2 / 15)\left(D_{\mathrm{W}} /\right.\right.$ $\left.\left.h_{\mathrm{c}}\right)^{9}+\left(D_{\mathrm{W}} / h_{\mathrm{c}}\right)^{3}\right]$, where the interaction strength $\epsilon_{\mathrm{W}}=2.0 \epsilon, h$ is the distance between the particle center and the wall, and $h_{\mathrm{c}}$ is the cutoff length of the potential. For the solvent beads, $D_{\mathrm{W}}=1 \sigma$ and $h_{\mathrm{c}}=3 \sigma$ $(0.8583 \sigma)$ at the lower (upper) wall. With these parameters, the liquid solvent completely wets the lower wall whereas the upper wall is purely repulsive. For the nanoparticles, both walls are repulsive with $D_{\mathrm{W}}=d / 2$ and $h_{\mathrm{c}}=0.8583 D_{\mathrm{W}}$, where $d$ is the nanoparticle diameter.

To model evaporation of the solvent, a rectangular box of dimensions $L_{x} \times L_{y} \times 20 \sigma$ at the top of the simulation cell is designated as a deletion zone and a certain number $(\zeta)$ of vapor beads of the solvent in this zone are removed every $\tau$, where $\tau=\sigma(\mathrm{m} / \epsilon)^{1 / 2}$ is the reduced $L J$ unit of time. In this paper, two evaporation rates $\zeta=$ 30 and 5 are adopted. At these rates, the liquid-vapor interface retreats during evaporation at almost a constant speed, $v_{\mathrm{e}}$. The value of $v_{\mathrm{e}}$ is determined for each evaporating suspension by directly computing the location of the interface as a function of time. The diffusion coefficients of nanoparticles are calculated with direct, independent simulations, and the results are $D_{1}=3.61 \times 10^{-3} \sigma^{2} / \tau$ for LNPs and $D_{\mathrm{s}}=2.11 \times 10^{-2} \sigma^{2} / \tau$ for SNPs at the initial volume fractions of nanoparticles prior to evaporation (see the Supporting Information). The ratio $D_{s} / D_{1}=5.8$ is higher than $\alpha=4$, the value expected from the Stokes-Einstein relation, which may be due to the finite concentrations of nanoparticles. ${ }^{38}$ With values of $D_{\mathrm{l}}, D_{\mathrm{s}}, v_{\mathrm{e}}$, and $H$ determined, the Péclet numbers for LNPs and SNPs, $\mathrm{Pe}_{1}$ and $\mathrm{Pe}_{s}$, are computed for each evaporating system.

The Large-scale Atomic/Molecular Massively Parallel Simulator ${ }^{39}$ (LAMMPS) was employed for all simulations reported here. A velocity-Verlet algorithm with a time step $\delta t=0.01 \tau$ was used to integrate the equation of motion. We have performed tests to confirm that the results reported here remain unchanged if a smaller time step is used. In the thermalized zone(s) specified for each system, a Langevin thermostat with a small damping rate $\Gamma=0.01 \tau^{-1}$ was used for the solvent beads. We have confirmed that this weak damping is strong enough to ensure a constant temperature in each thermalized liquid zone.

All results are presented below in LJ units. Here, we provide a rough mapping of these units to real ones in Table 1 by mapping the

Table 1. Rough Mapping between LJ and Real Units

\begin{tabular}{lll} 
physical quantity & LJ unit & \multicolumn{1}{c}{ SI value } \\
energy & $\epsilon$ & $7.6 \times 10^{-21} \mathrm{~J}$ \\
length & $\sigma$ & $0.35 \times 10^{-9} \mathrm{~m}$ \\
mass & $m$ & $4.5 \times 10^{-26} \mathrm{~kg}$ \\
time & $\tau$ & $0.85 \times 10^{-12} \mathrm{~s}$ \\
temperature & $\epsilon / k_{\mathrm{B}}$ & $550 \mathrm{~K}$ \\
velocity & $\sigma / \tau$ & $4.1 \times 10^{2} \mathrm{~m} / \mathrm{s}$ \\
diffusion coefficient & $\sigma^{2} / \tau$ & $1.4 \times 10^{-7} \mathrm{~m}^{2} / \mathrm{s}$ \\
density & $m / \sigma^{3}$ & $1.05 \times 10^{3} \mathrm{~kg} / \mathrm{m}^{3}$ \\
viscosity & $m /(\tau \sigma)$ & $1.5 \times 10^{-4} \mathrm{~Pa} \mathrm{~s}$ \\
pressure & $\epsilon / \sigma^{3}$ & $1.8 \times 10^{2} \mathrm{MPa}$ \\
\hline
\end{tabular}

LJ solvent adopted in this paper to a liquid with a critical point similar to that of water, a typical solvent used in drying experiments. ${ }^{7}$ The details of this mapping are provided in the Supporting Information.

\section{RESULTS AND DISCUSSION}

Our goal is to demonstrate that a temperature gradient and the associated thermophoretic effect can be used to control stratification in a drying suspension of a polydisperse mixture of nanoparticles. We have previously shown that particles of different sizes have different thermophoretic responses to a thermal gradient. ${ }^{30}$ In our previous work, only a thin layer of the liquid solvent adjacent to the bottom wall is thermalized at 
$T_{1}$ during evaporation, as shown in Figure 1a. Because of evaporative cooling at the liquid-vapor interface, a negative (a)

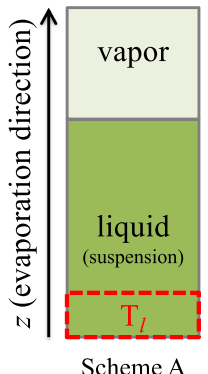

(b)

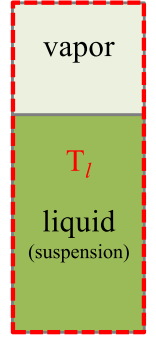

Scheme B (c)

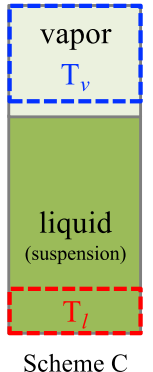

Figure 1. Schematics of three types of thermalizations during solvent evaporation: (a) Only a thin layer of the liquid solvent adjacent to the bottom wall is thermalized at $T_{1}$; (b) All liquid and vapor are thermalized at $T_{1}$; (c) A thin layer of the liquid solvent adjacent to the bottom wall is thermalized at $T_{1}$ whereas the vapor zone at some distance away from the equilibrium liquid-vapor interface is thermalized at $T_{\mathrm{v}}$. We set $T_{1}=1.0 \epsilon / k_{\mathrm{B}}$, and $T_{\mathrm{v}}$ can be higher or lower than $T_{1}$ to create a thermal gradient.

temperature gradient develops and its magnitude is larger for faster evaporation rates. The negative thermal gradient induces a positive gradient of the solvent density toward the interface, which generates a driving force to transport nanoparticles into the interfacial region. ${ }^{40,41}$ The thermophoretic driving force is stronger for larger particles. The Soret coefficient, $S_{\mathrm{T}}$, can be used to characterize the strength of thermophoretic motion with respect to diffusive motion of particles. We have performed independent simulations of thermophoresis at $A_{\text {ns }}$ $=100 \epsilon$ and found that for the LNPs, $S_{\mathrm{T}} \sim 0.1 \mathrm{~K}^{-1}$, whereas for the SNPs, their thermophoretic response is extremely weak and $S_{\mathrm{T}}$ is almost 0 (see the Supporting Information). As a result, for very fast evaporation, relatively more LNPs than SNPs are driven toward the interface in a drying bidisperse suspension. ${ }^{30}$ The thermophoresis caused by evaporative cooling competes with the diffusiophoresis that leads to small-on-top stratification at fast drying rates, which is why only weak small-on-top stratification was observed in our previous simulations. ${ }^{30}$ In certain cases the small-on-top stratification expected by the existing theory ${ }^{25}$ was even converted to large-on-top in the presence of strong evaporative cooling. ${ }^{30}$

On the basis of the physical picture depicted above, it is natural to investigate the effects of a controlled thermal gradient on stratification in a drying suspension. In this paper, we explore this idea by comparing three types of thermalization schemes, as sketched in Figure 1. Scheme A is the same as in our previous work in which only a $10 \sigma$ thick layer of the liquid solvent at the bottom of the suspension is thermalized at $T_{1}$ (Figure 1a). ${ }^{30}$ Evaporative cooling leads to a negative temperature gradient in the suspension toward the interface. In scheme $\mathrm{B}$, all solvent beads in the simulation cell are thermalized at $T_{1}$ (Figure $1 \mathrm{~b}$ ) and thus there are no thermal gradients during evaporation. In scheme $\mathrm{C}$, in addition to a liquid layer of thickness $10 \sigma$ thermalized at $T_{1}$ near the bottom wall, the vapor beads with $z$-coordinates between $L_{z}-150 \sigma$ and $L_{z}$ are coupled to a thermostat with a target temperature $T_{\mathrm{v}}$ (Figure 1c). In this way, a thermal gradient is generated in the suspension with its direction and magnitude controlled by the difference between $T_{\mathrm{v}}$ and $T_{\mathrm{v}}$, the thickness of the film, and the strength of evaporative cooling (i.e., the evaporation rate). For all systems studied in this paper, $T_{1}=1.0 \epsilon / k_{\mathrm{B}}$. For scheme C, $T_{\mathrm{v}}$ is varied from $0.75 \epsilon / k_{\mathrm{B}}$ to $1.2 \epsilon / k_{\mathrm{B}}$.

For scheme $A$, the systems are labeled as $T_{1.0}^{1} \zeta_{y}$ where the subscript $y$ denotes the value of $\zeta$. For scheme $B, T_{1.0} \zeta_{y}$ is used to emphasize that the entire system is maintained at $1.0 \epsilon / k_{\mathrm{B}}$ during evaporation. For scheme $\mathrm{C}$, the systems are labeled as $T_{1.0}^{\mathrm{l}} T_{x}^{\mathrm{v}} \zeta_{y}$, where $x$ indicates the value of $T_{\mathrm{v}}$. All systems studied are listed in Table 2. $T_{1.0}^{\mathrm{d}} T_{1.1}^{\mathrm{v}} \zeta_{5}, T_{1.0}^{\mathrm{l}} T_{1.05}^{\mathrm{v}} \zeta_{5}$, and $T_{1.0}^{\mathrm{l}} T_{1.0}^{\mathrm{v}} \zeta_{5}$ have

Table 2. Parameters for All Systems Studied ${ }^{a}$

\begin{tabular}{lrcrrl}
\multicolumn{1}{c}{ system } & $\zeta$ & $v_{\mathrm{e}} \tau / \sigma$ & $\mathrm{Pe}_{1}$ & $\mathrm{Pe}_{\mathrm{s}}$ & \multicolumn{1}{c}{$\begin{array}{c}\text { thermalization } \\
\text { scheme }\end{array}$} \\
$T_{1.0}^{\mathrm{l}} \zeta_{30}$ & 30 & $1.13 \times 10^{-3}$ & 95.2 & 16.3 & $\mathrm{~A}$ \\
$T_{1.0}^{1} \zeta_{5}$ & 5 & $2.04 \times 10^{-4}$ & 17.2 & 2.9 & $\mathrm{~A}$ \\
$T_{1.0} \zeta_{30}$ & 30 & $1.18 \times 10^{-3}$ & 99.4 & 17.0 & $\mathrm{~B}$ \\
$T_{1.0} \zeta_{5}$ & 5 & $2.11 \times 10^{-4}$ & 17.8 & 3.0 & $\mathrm{~B}$ \\
$T_{1.0}^{\mathrm{l}} T_{1.2}^{\mathrm{v}} \zeta_{5}$ & 5 & $2.04 \times 10^{-4}$ & 17.2 & 2.9 & $\mathrm{C}, T_{\mathrm{v}}=1.2 \epsilon / k_{\mathrm{B}}$ \\
$T_{1.0}^{\mathrm{l}} T_{1.1}^{\mathrm{v}} \zeta_{5}$ & 5 & $1.99 \times 10^{-4}$ & 16.8 & 2.9 & $\mathrm{C}, T_{\mathrm{v}}=1.1 \epsilon / k_{\mathrm{B}}$ \\
$T_{1.0}^{1} T_{1.05}^{\mathrm{v}} \zeta_{5}$ & 5 & $2.04 \times 10^{-4}$ & 17.2 & 2.9 & $\mathrm{C}, T_{\mathrm{v}}=1.05 \epsilon / k_{\mathrm{B}}$ \\
$T_{1.0}^{\mathrm{l}} T_{1.0}^{\mathrm{v}} \zeta_{5}$ & 5 & $2.07 \times 10^{-4}$ & 17.4 & 3.0 & $\mathrm{C}, T_{\mathrm{v}}=1.0 \epsilon / k_{\mathrm{B}}$ \\
$T_{1.0}^{\mathrm{1}} T_{0.9}^{\mathrm{v}} \zeta_{5}$ & 5 & $6.93 \times 10^{-4}$ & 58.4 & 10.0 & $\mathrm{C}, T_{\mathrm{v}}=0.9 \epsilon / k_{\mathrm{B}}$ \\
$T_{1.0}^{\mathrm{l}} T_{0.85}^{\mathrm{v}} \zeta_{5}$ & 5 & $9.90 \times 10^{-4}$ & 83.4 & 14.3 & $\mathrm{C}, T_{\mathrm{v}}=0.85 \epsilon / k_{\mathrm{B}}$ \\
$T_{1.0}^{\mathrm{l}} T_{0.75}^{\mathrm{v}} \zeta_{5}$ & 5 & $1.03 \times 10^{-3}$ & 86.7 & 14.8 & $\mathrm{C}, T_{\mathrm{v}}=0.75 \epsilon / k_{\mathrm{B}}$
\end{tabular}

${ }^{a}$ Refer to Figure 1 for the thermalization schemes.

results in line with $T_{1.0}^{\mathrm{l}} T_{1.2}^{\mathrm{v}} \zeta_{5}$. We also studied systems with $\zeta=$ 5 and $T_{\mathrm{v}}<T_{1}$, which show negative thermal gradients in the suspension and thermophoresis similar to those in $T_{1.0}^{1} \zeta_{30}$ and $T_{1.0}^{1} \zeta_{5}$ where evaporative cooling occurs. However, we observed condensation of droplets in the vapor phase if $T_{\mathrm{v}}$ is made lower than the temperature at the liquid-vapor interface in scheme A with the same $\zeta$. Despite this unwanted effect, cooling the vapor at a temperature lower than that of the suspension could be one experimental approach to apply a negative thermal gradient for systems that evaporate slowly or for which the effect of evaporative cooling is not as strong as that for the model LJ liquid employed in our simulations. The last six systems in Table 2 with $T_{\mathrm{v}}$ varying from $0.75 \epsilon / k_{\mathrm{B}}$ to $1.1 \epsilon / k_{\mathrm{B}}$ are included in the Supporting Information. In the main text, we focus on the first five systems in Table 2 .

Snapshots of the first five nanoparticle suspensions in Table 2 during solvent evaporation are shown in Figure 2. For $T_{1.0}^{\mathrm{l}} \zeta_{30}$ and $T_{1.0}^{1} \zeta_{5}$ (Figure 2a,b), the evaporative cooling of the liquidvapor interface leads to a negative thermal gradient along the normal direction toward the interface. Although for both systems small-on-top stratification is expected by the model of Zhou et al. since $\mathrm{Pe}_{1} \gg \mathrm{Pe}_{\mathrm{s}}>1,{ }^{20}$ the thermophoresis associated with the negative temperature gradient works against diffusiophoresis and transports more LNPs into the interfacial region. As a result, the two systems exhibit large-ontop stratification.

When all solvent beads in the simulation cell are thermalized during evaporation, the temperature in the entire system is constant and no thermal gradients are produced. Thermophoresis is thus suppressed, and only diffusiophoresis remains. The expected outcome is small-on-top stratification for $\mathrm{Pe}_{1} \gg \mathrm{Pe}_{\mathrm{s}}>$ 1. The results from $T_{1.0} \zeta_{30}$ and $T_{1.0} \zeta_{5}$ confirm this prediction, as shown in Figure $2 \mathrm{c}, \mathrm{d}$. For example, comparing the last snapshot for $T_{1.00_{5}}^{1}$ (the second row of Figure 2) and that for $T_{1.0} \zeta_{5}$ (the fourth row of Figure 2), the transition from largeon-top to small-on-top is visible after the thermal gradients and 

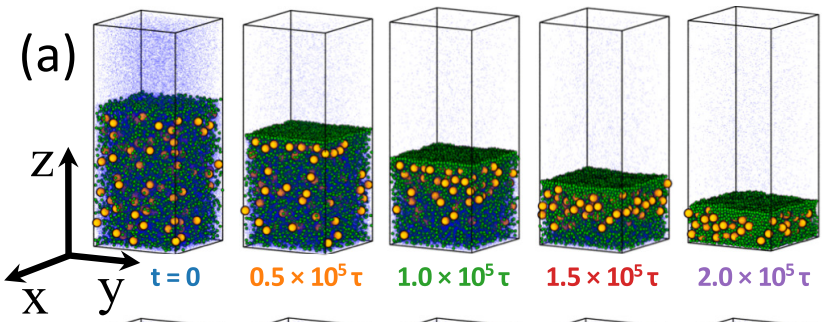

(b)
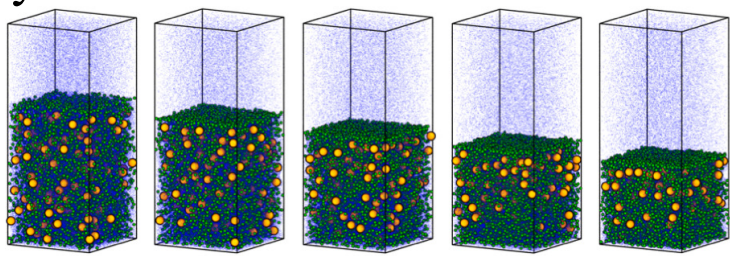

(c)
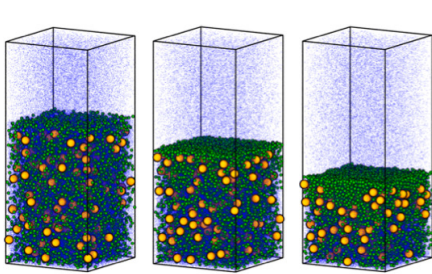

$6.0 \times 10^{5} \tau$
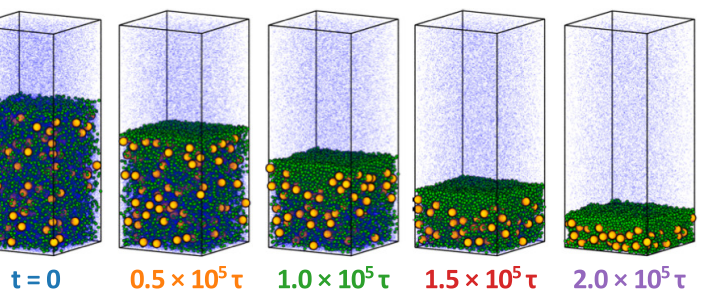

(d)
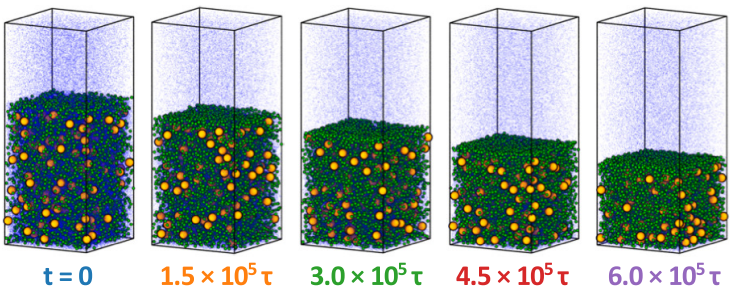

(e)
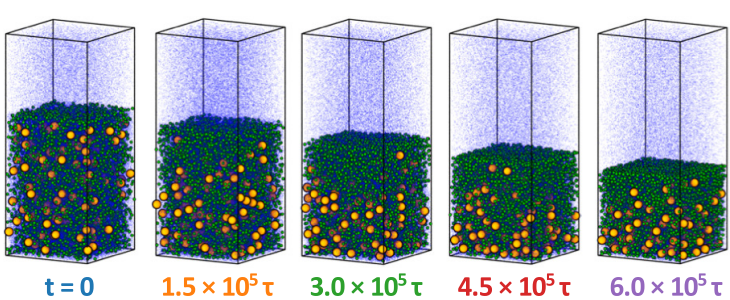

Figure 2. Snapshots during solvent evaporation for (a) $T_{1.0}^{1} \zeta_{30}$, (b) $T_{1.0}^{\mathrm{l}} \zeta_{5}$, (c) $T_{1.0} \zeta_{30}$, (d) $T_{1.0} \zeta_{5}$, and (e) $T_{1.0}^{\mathrm{l}} T_{1.2}^{\mathrm{v}} \zeta_{5}$. The elapsed time since the initiation of evaporation at $t=0$ is listed under each snapshot. Temperature and density profiles of the five systems are shown in Figure 3. Color code: SNPs (green), LNPs (orange), and solvent (blue). Only $5 \%$ of the solvent beads are visualized to improve clarity.

the associated thermophoresis are inhibited, especially from the distribution of LNPs in the drying films. This transition is verified quantitatively by an order parameter of stratification, which is discussed below (see Figure 4).

The last row of Figure 2 shows the snapshots for $T_{1.0}^{\mathrm{l}} T_{1.2}^{\mathrm{v}} \zeta_{5}$. In this system, the vapor at about $23 \sigma$ above the initial liquidvapor interface prior to evaporation is thermalized at $T_{\mathrm{v}}=$ $1.2 \epsilon / k_{\mathrm{B}}>T_{1}$ during evaporation. Consequently, there is a positive temperature gradient in the liquid solvent along the film's normal direction toward the interface. The solvent density develops a negative gradient and the accompanied thermophoresis drives LNPs toward the substrate. As a result, thermophoretic and diffusiophoretic effects are in synergy and strong small-on-top stratification is generated, which is apparent in Figure 2e where the LNPs are enriched near the substrate during drying.

To understand quantitatively the stratification phenomena in drying particle suspensions, we plot the temperature and density profiles in Figure 3. The local temperature $T(z)$ at height $z$ is computed from the average kinetic energy of the solvent beads in the spatial bin $[z-2.5 \sigma, z+2.5 \sigma] .^{42}$ The temperature profiles in the top row of Figure 3 clearly show the negative thermal gradients induced by evaporative cooling for $T_{1.0}^{\mathrm{l}} \zeta_{30}$ and $T_{1.0}^{\mathrm{l}} \zeta_{5}$, with the effect stronger at larger evaporation rates. $T_{1.0} \zeta_{30}$ and $T_{1.0} \zeta_{5}$ do not exhibit thermal gradients as all of the solvent is thermalized at $T_{1}$, as shown in Figure 3i,m. $T_{1.0}^{\mathrm{l}} T_{1.2}^{\mathrm{v}} \zeta_{5}$ with $T_{\mathrm{v}}>T_{1}$ exhibits an externally imposed positive thermal gradient (Figure 3q).

The local density of solvent or nanoparticles is computed as $\rho_{i}(z)=n_{i}(z) m_{i} /\left(L_{x} L_{y} \sigma\right)$, where $n_{i}(z)$ represents the number of particles in the spatial bin $[z-0.5 \sigma, z+0.5 \sigma]$ and $m_{i}$ is the particle mass. A nanoparticle straddling several bins is partitioned on the basis of its partial volume in each bin. When computing the solvent density, the volume occupied by the nanoparticles is subtracted. The second row of Figure 3 shows the solvent density as a function of height, and the profiles exhibit gradients in accordance with the thermal gradients. Particularly, a positive (negative) thermal gradient generates a negative (positive) density gradient for the solvent and the stronger the thermal gradient, the stronger the density gradient. This correlation results from the fact that local thermal equilibrium is always maintained even at the fastest evaporation rates adopted in our simulations. ${ }^{42}$ The density profile of the solvent affects the receding speed, $v_{\mathrm{e}}$, of the liquid-vapor interface. The data in Table 2 show that at the same $\zeta$, the value of $v_{\mathrm{e}}$ is slightly lower for $T_{1.0}^{\mathrm{l}} \zeta_{y}$ under scheme A than for $T_{1.0} \zeta_{y}$ under scheme B. For $T_{1.0}^{1} \zeta_{y}$, the evaporative cooling causes a positive gradient of the solvent density. The average solvent density is thus higher for $T_{1.0}^{1} \zeta_{y}$ than for $T_{1.0} \zeta_{y}$, as shown in Figure $3 b, f, j, n$. As a result, the liquid-vapor interface recedes more slowly in $T_{1.0}^{1} \zeta_{y}$ than in $T_{1.0} \zeta_{y}$ at the same $\zeta$

The density profiles for LNPs and SNPs are shown in the bottom two rows of Figure 3, respectively. These profiles demonstrate the phoretic response of the nanoparticles to the thermal gradients (equivalently, the density gradients of the solvent induced by the thermal gradients) as well as the effects of the evaporation rate. For all simulations discussed here, the evaporation rates are high enough such that $\mathrm{Pe}_{1} \gg \mathrm{Pe}_{\mathrm{s}}>1$. The corresponding fast receding liquid-vapor interface tends to trap both LNPs and SNPs just below the interface. If no other factors are at play, this effect combined with a large enough $\phi_{\mathrm{s}}$ is expected to yield small-on-top stratification via the diffusiophoresis mechanism, as suggested by Sear and collaborators $^{18,25}$ and Zhou et al. ${ }^{20}$ This scenario is indeed the case for $T_{1.0} \zeta_{30}$ and $T_{1.0} \zeta_{5}$, as shown in the third and fourth columns of Figure 3 where there are no thermal gradients. The diffusiophoresis model also implies that the degree of small-ontop stratification is enhanced when the evaporation rate is increased. ${ }^{18,20}$ However, as shown later, $T_{1.0} \zeta_{5}$ actually exhibits stronger small-on-top stratification than $T_{1.0} \zeta_{30}$, even though the evaporation rate is increased 6-fold in the latter system. This discrepancy may be partially due to the small thickness of the suspension film studied in our simulations, which is limited by the available computational resources. The effect of film thickness on stratification is explored in a separate study. ${ }^{43}$ Another reason may be that when the evaporation rate is 

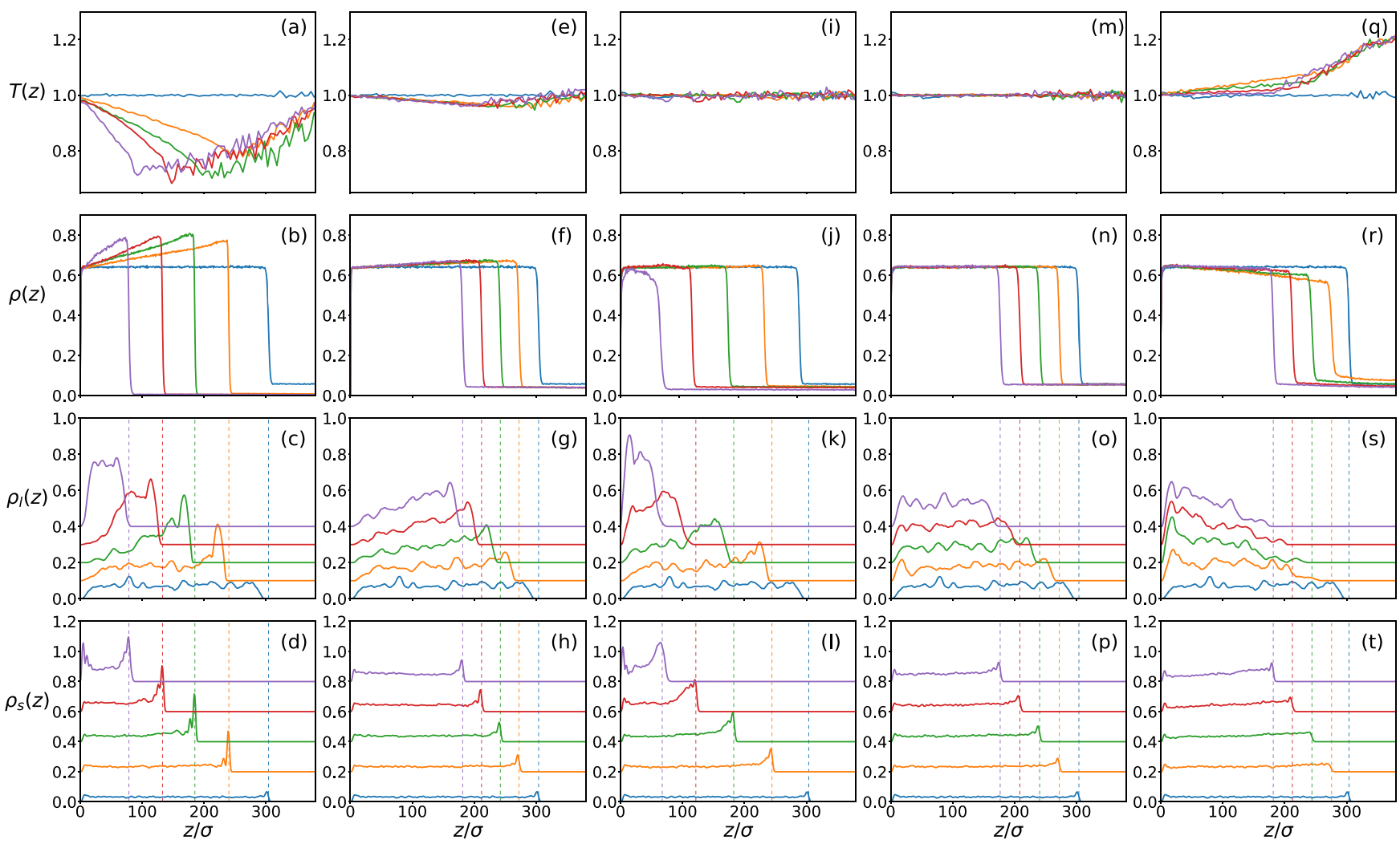

Figure 3. Temperature profiles (top row) and density profiles for the solvent (second row), LNPs (third row), and SNPs (bottom row) for $T_{1.0}^{1} \zeta_{30}$ $(\mathrm{a}-\mathrm{d}), T_{1.0}^{\mathrm{l}} \zeta_{5}(\mathrm{e}-\mathrm{h}), T_{1.0} \zeta_{30}(\mathrm{i}-\mathrm{l}), T_{1.0} \zeta_{5}(\mathrm{~m}-\mathrm{p})$, and $T_{1.0}^{\mathrm{l}} T_{1.2}^{\mathrm{v}} \zeta_{5}(\mathrm{q}-\mathrm{t})$, respectively. The curves follow the same order as the snapshots shown in Figure 2. The vertical dashed lines indicate the location of the liquid-vapor interface. For clarity, the density profiles for LNPs (SNPs) are shifted upward by $0.1 \mathrm{~m} / \sigma^{3}\left(0.2 \mathrm{~m} / \sigma^{3}\right)$ successively.

increased, the drying time is shortened and there is less time for LNPs to diffuse out of the interfacial region via diffusiophoresis. As a result, small-on-top stratification is weakened when the evaporation rate is enhanced beyond a certain threshold. This trend indicates that small-on-top stratification is most enhanced at some $\mathrm{Pe}_{1}$ and is diminished if $\mathrm{Pe}_{1}$ is increased further, which is consistent with two recent reports. $^{28,43}$

When only a thin layer of solvent beads at the bottom wall is thermalized, the temperature in the vicinity of the liquidvapor interface decreases because of evaporative cooling effect. The resulting enhancement of the solvent density at the interface leads to thermophoretic drift of nanoparticles with the effect more significant for larger particles. This physical picture explains the observations for $T_{1.0}^{1} \zeta_{30}$ and $T_{1.0}^{1} \zeta_{5}$. In these two systems, the SNPs are found to accumulate at the surface of the evaporating suspension as $\mathrm{Pe}_{1} \gg \mathrm{Pe}_{\mathrm{s}}>1$ (Figure 3d,h). However, a significant accumulation of LNPs is found just below the enriched surface layer of SNPs, as shown in Figure $3 \mathrm{c}, \mathrm{g}$. The net outcome is actually large-on-top stratification, which will be confirmed later with an order parameter quantifying stratification (see Figure 4). Furthermore, the degree of large-on-top stratification is stronger for $T_{1.0 \zeta_{5}}^{1}$ than for $T_{1.0}^{1} \zeta_{30}$, indicating a delicate competition between diffusiophoresis and thermophoresis. The lower evaporation rate in $T_{1.0}^{1} \zeta_{5}$ suppresses both processes, but it appears that diffusiophoresis favoring small-on-top is mitigated slightly more, creating stronger large-on-top for $T_{1.0}^{1} \zeta_{5}$.

In our previous work, ${ }^{30}$ we obtained a state diagram of stratification with all systems thermalized with scheme A (i.e., a

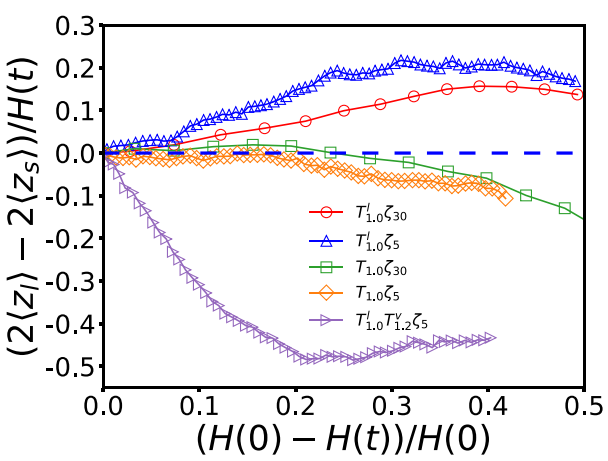

Figure 4. Mean separation between LNPs and SNPs normalized by $H(t) / 2$, vs extent of drying, $(H(0)-H(t)) / H(0)$, for $T_{1.0}^{1} \zeta_{30}$ (red circle), $T_{1.0}^{\mathrm{l}} \zeta_{5}$ (blue upward triangle), $T_{1.0} \zeta_{30}$ (green square), $T_{1.0} \zeta_{5}$ (yellow diamond), and $T_{1.0}^{\mathrm{l}} T_{1.2}^{\mathrm{v}} \zeta_{5}$ (purple right-pointing triangle).

thin layer of liquid solvent contacting the substrate is thermalized at $\left.T_{1}=1.0 \epsilon / k_{\mathrm{B}}\right)$ and only observed weak smallon-top stratification at values of $\mathrm{Pe}_{\mathrm{s}}$ and $\phi_{\mathrm{s}}$ far exceeding the critical values predicted by the diffusiophoretic model of Zhou et al. ${ }^{20}$ The presence of thermophoresis at fast evaporation rates may help understand the discrepancy between the simulations and the theory. ${ }^{30}$ Indeed, when thermophoresis is suppressed, systems that are driven into the large-on-top regime by thermophoresis can be turned into (usually weak) small-on-top. Examples are the transition from $T_{1.0}^{1} \zeta_{30}$ to $T_{1.0} \zeta_{30}$ and that from $T_{1.0}^{1} \zeta_{5}$ to $T_{1.0} \zeta_{5}$.

To achieve strong small-on-top stratification, a natural idea is to enable thermophoresis that works in conjunction with 
diffusiophoresis. This cooperation requires a thermal gradient during evaporation that is opposite to the one induced by evaporative cooling. To realize this, we thermalize the vapor zone from $L_{z}-150 \sigma$ to $L_{z}$ at a temperature $T_{\mathrm{v}}>T_{1}$. The data in the fifth column of Figure 3 are for $T_{1.0}^{\mathrm{l}} T_{1.2}^{\mathrm{v}} \zeta_{5}$ where $T_{\mathrm{v}}=$ $1.2 \epsilon / k_{\mathrm{B}}$. A positive thermal gradient and a negative density gradient of the solvent can be seen clearly in Figure 3q,r, respectively. Since the gradients are reversed, the LNPs are now driven toward the substrate via thermophoresis (Figure $3 s$ ) whereas the SNPs are much less affected (Figure 3t). The final result is strong small-on-top stratification where the LNPs are accumulated near the substrate and depleted in the interfacial region whereas the SNPs exhibit a positive density gradient (i.e., accumulation) from the bulk of the film to the receding interface as $\mathrm{Pe}_{\mathrm{s}}>1$.

It is expected that for systems thermalized with scheme $\mathrm{C}$ and $T_{\mathrm{v}}<T_{1}$, a negative thermal gradient develops in the liquid solvent, similar to the evaporative cooling case in scheme A. Consequently, systems under scheme $\mathrm{C}$ with $T_{\mathrm{v}}<T_{1}$ could display large-on-top stratification as long as the thermal gradient is large enough. These cases are in fact observed and discussed in detail in the Supporting Information, where some complications are noted related to droplet condensation in vapor that is thermalized at low temperatures. Even for $T_{\mathrm{v}} \gtrsim$ $T_{1}$, the thermal gradient in the drying suspension can still be negative if evaporative cooling is strong enough. This is the case for $T_{1.0}^{\mathrm{l}} T_{1.05}^{\mathrm{v}} \zeta_{5}$ and $T_{1.0}^{\mathrm{l}} T_{1.0}^{\mathrm{v}} \zeta_{5}$ (see the Supporting Information).

To quantify stratification, we define an order parameter using the full density profiles of nanoparticles. ${ }^{30}$ The mean heights of LNPs and SNPs are computed as $\left\langle z_{i}\right\rangle=\frac{1}{N_{i}} \sum_{n=1}^{N_{i}} z_{\text {in }}$ with $i \in\{1, s\}$. The order parameter of stratification is then computed as $\left(2\left\langle z_{1}\right\rangle-2\left\langle z_{\mathrm{s}}\right\rangle\right) / H(t)$, i.e., the mean separation between LNPs and SNPs, normalized by $H(t) / 2$, where $H(t)$ is the instantaneous thickness of the suspension. In the equilibrium suspension prior to evaporation, both $\left\langle z_{1}\right\rangle$ and $\left\langle z_{\mathrm{s}}\right\rangle$ are very close to $H(0) / 2$, where $H(0)$ is the initial film thickness. During evaporation, $\left\langle z_{1}\right\rangle-\left\langle z_{s}\right\rangle<0$ indicates smallon-top stratification whereas $\left\langle z_{1}\right\rangle-\left\langle z_{\mathrm{s}}\right\rangle>0$ signifies large-ontop.

In Figure 4, the order parameter of stratification is plotted against the extent of drying, quantified as $(H(0)-H(t))$ / $H(0)$, for the first five systems listed in Table 2 . It is clear that $T_{1.0} \zeta_{30}$ and $T_{1.0} \zeta_{5}$ exhibit small-on-top stratification since diffusiophoresis dominates whereas thermal gradients and thermophoresis are absent. The extent of stratification is slightly stronger for $T_{1.0} \zeta_{5}$, although it dries more slowly. Large-on-top is observed for $T_{1.0}^{1} \zeta_{30}$ and $T_{1.0}^{1} \zeta_{5}$ and is again stronger for $T_{1.0}^{1} \zeta_{5}$ that has a smaller evaporation rate. Although thermophoresis is much weaker for $T_{1.0}^{1} \zeta_{5}$ because of the reduced evaporation rate, diffusiophoresis favoring small-on-top is suppressed even more when evaporation is slowed down and the delicate interplay of the two phoretic processes leads to stronger large-on-top stratification for $T_{1.0}^{1} \zeta_{5}$.

A dramatic small-on-top state is clearly demonstrated in Figure 4 for $T_{1.0}^{\mathrm{l}} T_{1.2}^{\mathrm{v}} \zeta_{5}$. Note that in the equilibrium suspension, $\phi_{1}=2 \phi_{\mathrm{s}}$. If in the final dry film, all SNPs were on top of all LNPs (i.e., complete stratification) but each group is uniformly distributed in its own region, then $\left\langle z_{1}\right\rangle=H(t) / 3$ and $\left\langle z_{\mathrm{s}}\right\rangle=$ $5 H(t) / 6$, yielding $\left(2\left\langle z_{1}\right\rangle-2\left\langle z_{s}\right\rangle\right) / H(t)=-1$. As shown in Figure 4 , the order parameter of stratification reaches a minimal value at around -0.5 for $T_{1.0}^{\mathrm{l}} T_{1.2}^{\mathrm{v}} \zeta_{5}$, indicating that the vertical distribution of the binary mixture of nanoparticles is substantially segregated in the drying film with SNPs on top of LNPs. This outcome is visually apparent in Figure $2 \mathrm{e}$ as well.

The stratification order parameter used here and in ref 30 is based on the average position of nanoparticles, which is the first moment of their density profile in the entire drying film. This order parameter describes the systems studied here well, and the identification of a stratified state is consistent with the classification based on the overall trend of the density profile of nanoparticles in the bulk of the drying film. Namely, small-ontop stratification generally corresponds to a negative gradient of the density profile of LNPs and a positive or nearly zero gradient of the density profile of SNPs from the bottom of the film to the top, whereas large-on-top is the other way around. However, this order parameter may not be applicable to oscillating density profiles or systems with only local stratification. In these more complicated situations, some other characteristics of the nanoparticle distribution including the higher moments of the density profile may be necessary to classify stratification. For all of our simulations, there is always a layer enriched with SNPs at the top of the drying film because $\mathrm{Pe}_{\mathrm{s}}>1$. However, it is misleading to call all of these systems small-on-top. Instead, information on the nanoparticle distribution in the film below this SNP-rich surface layer should be taken into account as well. The order parameter used here fulfills this goal and yields a more consistent classification scheme for the outcome of stratification.

Evaporative cooling is a natural effect in a fast drying liquid. If a particle suspension is placed on a substrate that is kept at a constant temperature and the suspension undergoes very fast solvent evaporation, then a temperature lower than that of the substrate is expected at the evaporating interface, resulting in a negative thermal gradient in the suspension. $T_{1.0}^{1} \zeta_{30}$ and $T_{1.0}^{1} \zeta_{5}$ studied here are set up to mimic such situations. However, it is challenging to maintain a constant temperature or induce a positive thermal gradient along the normal direction toward the interface in a drying suspension, especially when the evaporation rate is high. One possible approach is to dissolve a gas (e.g., $\mathrm{N}_{2}, \mathrm{Ar}, \mathrm{He}$, or $\mathrm{CO}_{2}$ ) into the solvent (e.g., water). Beaglehole showed that heating a water film with a dissolved gas from above or below produces very different temperature distributions within the liquid. ${ }^{44,45}$ When heated from below, a fairly uniform temperature is found throughout the liquid. However, when the liquid is heated from above, a temperature gradient develops in it with the temperature higher at the liquid-vapor interface. Then, it may be possible to study the effect of solvent evaporation on the particle distribution in a drying film under isothermal conditions and positive thermal gradients, similar to schemes $\mathrm{B}$ and $\mathrm{C}$.

In most experiments, films are much thicker than those studied here with $\mathrm{MD}$ and evaporation rates are much lower by a factor of $10^{4}-10^{5}$ for drying at room temperature, about $45 \%$ of the critical temperature of water. In these systems, evaporative cooling is negligible and heat transfer is fast enough to make temperature uniform throughout a drying film. ${ }^{46,47}$ To mimic this situation, scheme B is used to maintain an isothermal drying film by coupling all solvent beads including vapor to a weak Langevin thermostat with a small damping rate, $\Gamma=0.01 \tau^{-1}$. To address whether hydrodynamic interactions are screened in Langevin dynamics, ${ }^{48}$ we run an additional simulation for $T_{1.0} \zeta_{30}$ with the Langevin thermostat replaced by a pairwise thermostat based on dissipative particle dynamics (DPD), with a weak friction coefficient $\gamma=0.05 \mathrm{~m} /$ 
$\tau .^{49}$ With the DPD thermostat, local momentum conservation is preserved throughout the simulation box and hydrodynamic interactions are expected to be fully captured. The results with the DPD thermostat are very close to those discussed here with the Langevin thermostat. These results are included in the Supporting Information. Under scheme A and C, local momentum conservation is fulfilled away from the thermalized zones. All tests indicate that the Langevin thermostat adopted here is weak enough such that the viscosity of the LJ liquid is only weakly altered and the screening effect on hydrodynamic interactions is negligible.

In all simulations discussed thus far, the temperature of the thermalized liquid zone is always $1.0 \epsilon / k_{\mathrm{B}}$. The highest temperature used for the thermalized vapor zone is $1.2 \epsilon / k_{\mathrm{B}}$, which is close to the critical temperature, $T_{\mathcal{O}}$ of the $\mathrm{LJ}$ solvent with $r_{\mathrm{c}}=3.0 \sigma$. Furthermore, all simulations start with systems in which the nanoparticles are uniformly dispersed and then the evaporation process and the thermal gradient are imposed simultaneously. With this approach, the interplay between diffusiophoresis and thermophoresis is investigated. To ensure that the physical mechanism of controlling stratification via thermophoresis is applicable to systems with both liquid and vapor temperatures way below $T_{\mathcal{c}}$, we run an additional simulation for $R T_{0.9}^{\mathrm{l}} T_{1.0}^{\mathrm{v}} \zeta_{5}$, i.e., with the bottom layer of the solvent adjacent to the lower wall thermalized at $0.9 \epsilon / k_{\mathrm{B}}$ while the vapor zone above the liquid-vapor interface is thermalized at $1.0 \epsilon / k_{\mathrm{B}}$. For $R T_{0.9}^{1} T_{1.0}^{\mathrm{v}} \zeta_{5}$, the system is first relaxed under the imposed thermal gradient, which causes the LNPs to drift toward the lower wall via thermophoresis. The SNPs are still uniformly dispersed in the film as they are almost irresponsive to the thermal gradient. Then, the solvent is evaporated from the relaxed system. The results for $R T_{0.9}^{1} T_{1.0}^{\mathrm{v}} \zeta_{5}$ are discussed in detail in the Supporting Information and fully consistent with the idea that thermophoresis from a positive thermal gradient from the bulk of a film to its drying front works in synergy with diffusiophoresis to enhance small-on-top, whereas a negative thermal gradient works against diffusiophoresis to suppress small-on-top and promote large-on-top.

\section{CONCLUSIONS}

In this paper, we focus on how stratification can be controlled in a drying suspension of a bidisperse mixture of nanoparticles via $\mathrm{MD}$ simulations with an explicit solvent model. We demonstrate that a thermal gradient and the induced thermophoresis can be used to alter stratification from largeon-top all the way to strong small-on-top. This strategy is based on the observation that particles of different sizes in a suspension have different responses to a thermal gradient. In particular, larger particles experience a larger driving force that transports them into cooler regions where the solvent density is higher. For $A_{\mathrm{ns}}=100 \epsilon$ adopted here, the smaller nanoparticles show little or even no response to a thermal gradient. When a suspension undergoes fast drying and only a thin layer of the solvent adjacent to the substrate is thermalized at $T_{\mathrm{b}}$, mimicking an experimental situation where the substrate supporting the suspension is maintained at a constant temperature during solvent evaporation, a negative temperature gradient develops in the suspension because of the evaporative cooling effect that makes the temperature at the evaporating interface to drop below $T_{1}$. A larger fraction of the larger nanoparticles is driven into the interfacial region via the thermophoresis induced by this thermal gradient. As a result, the fast drying suspensions display large-on-top stratification instead of small-on-top expected by the diffusiophoresis model in which the suspension is assumed to be isothermal during evaporation. ${ }^{18,20,25}$

Interestingly, when the entire suspension is maintained at $T_{1}$ during drying by thermalizing all solvent beads in the simulation cell, they do exhibit small-on-top stratification at fast evaporation rates, consistent with the prediction of the diffusiophoresis model. ${ }^{18,20,25}$ However, the degree of stratification is found to be weak, probably due to the fact that $\phi_{\mathrm{s}}$ is small and the liquid film is thin for the simulations reported here. When a positive thermal gradient is induced in the suspension by thermalizing the vapor at a temperature sufficiently higher than $T_{1}$, all larger nanoparticles are propelled toward the substrate. In this case, the synergy between thermophoresis and diffusiophoresis underlies the observation of strong small-on-top stratification. Our results thus reveal a potentially useful strategy of controlling stratification via a regulated thermal gradient in a drying suspension of polydisperse particles.

The film thickness in our simulations prior to evaporation is about $300 \sigma \sim 105 \mathrm{~nm}$. For a temperature difference of $0.1 \epsilon / k_{\mathrm{B}}$ across the film, the magnitude of the thermal gradient is about $0.5 \mathrm{~K} / \mathrm{nm}$ if we take $\epsilon / k_{\mathrm{B}} \sim 550 \mathrm{~K}$, as in Table 1 . This thermal gradient is several orders of magnitude larger than a typical experimental value. However, the suspensions simulated here are at temperatures not far from the critical temperature of the solvent, which allows the evaporation process of the solvent to be fast enough that can be modeled with MD. As a result, the evaporation rates in the $\mathrm{MD}$ simulations are also much higher than those in experiments. Nevertheless, as already discussed in ref 30, such high evaporation rates are needed to drive a submicron thin-film suspension of nanoparticles into the "small-on-stop" regime (i.e., $\mathrm{Pe}_{\mathrm{s}}>1$ ), demonstrated in silico with our simulations. It is an interesting challenge if such a scenario can be realized experimentally, for example, by bringing the suspension close to the critical point of its solvent, as it may be relevant to the fabrication of multilayered thin-film coatings. Because of high evaporation rates and the resulting strong evaporative cooling, large thermal gradients are needed to control stratification in drying thin films. For films with micrometer to millimeter thickness as in many experiments, ${ }^{7}$ evaporation rates and thermal gradients are smaller by a factor of $10^{4}-10^{5}$ than those employed in MD simulations, i.e., those within the typical experimental range, will be sufficient to drive the systems into the same physical regime where thermophoresis is comparable to diffusiophoresis. In this sense, the results obtained here from MD simulations with thin films are scalable to much thicker films studied in experiments.

\section{ASSOCIATED CONTENT}

\section{Supporting Information}

The Supporting Information is available free of charge on the ACS Publications website at DOI: 10.1021/acs.langmuir.8b03659.

Mean square displacement vs time for nanoparticles; different thermophoretic responses of LNPs and SNPs; snapshots and density profiles for $T_{1.0}^{\mathrm{l}} T_{1.1}^{\mathrm{v}} \zeta_{5}, T_{1.0}^{\mathrm{l}} T_{1.05}^{\mathrm{v}} \zeta_{5}$, $T_{1.0}^{\mathrm{l}} T_{1.0}^{\mathrm{v}} \zeta_{5}$, and $T_{1.0}^{\mathrm{l}} T_{0.75}^{\mathrm{v}} \zeta_{5}$; average position of nanoparticles and their average separation as a function of time for $T_{1.0}^{\mathrm{l}} T_{1.0}^{\mathrm{v}} \zeta_{5}, T_{1.0}^{\mathrm{l}} T_{1.05}^{\mathrm{v}} \zeta_{5}, T_{1.0}^{\mathrm{l}} T_{1.1}^{\mathrm{v}} \zeta_{5}$, and $T_{1.0}^{\mathrm{l}} T_{1.2}^{\mathrm{v}} \zeta_{5}$; average position of nanoparticles as a function of time for $T_{1.0}^{1} \zeta_{30}, \quad T_{1.0}^{1} \zeta_{5}, \quad T_{1.0} \zeta_{30}, \quad T_{1.0} \zeta_{5}, \quad T_{1.0}^{1} T_{1.2}^{\mathrm{v}} \zeta_{5}$, and 
$T_{1.0}^{\mathrm{l}} T_{0.75}^{\mathrm{v}} \zeta_{5} ;$ average separation between LNPs and SNPs as a function of time for $T_{1.0}^{\mathrm{1}} \zeta_{30}, T_{1.0}^{\mathrm{1}} \zeta_{5}$, and $T_{1.0}^{\mathrm{1}} T_{0.75}^{\mathrm{v}} \zeta_{5}$; average position of nanoparticles and their average separation as a function of time for $T_{1.0}^{1} T_{0.75}^{\mathrm{v}} \zeta_{5}$, $T_{1.0}^{\mathrm{l}} T_{0.85}^{\mathrm{v}} \zeta_{5}$, and $T_{1.0}^{\mathrm{1}} T_{0.9}^{\mathrm{v}} \zeta_{5}$; snapshots, density profiles, and average position of nanoparticles and their average separation as a function of time for $R T_{0.9}^{\mathrm{l}} T_{1.0}^{\mathrm{v}} \zeta_{5}$; snapshots and density profiles for $T_{1.0} \zeta_{5}$ with a thermostat based on dissipative particle dynamics (DPD); comparison of average position of nanoparticles and their average separation as a function of time from $T_{1.0} \zeta_{5}$ with the Langevin thermostat to those from $T_{1.0} \zeta_{5}$ with the DPD thermostat (PDF)

\section{AUTHOR INFORMATION}

\section{Corresponding Author}

*E-mail: chengsf@vt.edu.

\section{ORCID $\odot$}

Gary S. Grest: 0000-0002-5260-9788

Shengfeng Cheng: 0000-0002-6066-2968

\section{Notes}

The authors declare no competing financial interest.

\section{ACKNOWLEDGMENTS}

Acknowledgement is made to the Donors of the American Chemical Society Petroleum Research Fund (PRF \#56103DNI6) for support of this research. This research used resources of the National Energy Research Scientific Computing Center (NERSC), a U.S. Department of Energy Office of Science User Facility operated under Contract no. DE-AC02-05CH11231. These resources were obtained through the Advanced Scientific Computing Research (ASCR) Leadership Computing Challenge (ALCC). This work was performed, in part, at the Center for Integrated Nanotechnologies, an Office of Science User Facility operated for the U.S. Department of Energy Office of Science. Sandia National Laboratories is a multimission laboratory managed and operated by National Technology and Engineering Solutions of Sandia, LLC., a wholly owned subsidiary of Honeywell International, Inc., for the U.S. Department of Energy's National Nuclear Security Administration under contract DE-NA0003525. This paper describes objective technical results and analysis. Any subjective views or opinions that might be expressed in the paper do not necessarily represent the views of the U.S. Department of Energy or the United States Government.

\section{REFERENCES}

(1) Sheetz, D. P. Formation of films by drying of latex. J. Appl. Polym. Sci. 1965, 9, 3759-3773.

(2) Keddie, J. L. Film formation of latex. Mater. Sci. Eng., R 1997, 21, 101-170.

(3) Steward, P. A.; Hearn, J.; Wilkinson, M. An overview of polymer latex film formation and properties. Adv. Colloid Interface Sci. 2000, $86,195-267$.

(4) Russel, W. B. Mechanics of drying colloidal dispersions: Fluid/ solid transitions, skinning, crystallization, cracking, and peeling. AIChE J. 2011, 57, 1378-1385.

(5) Routh, A. F. Drying of thin colloidal films. Rep. Prog. Phys. 2013, 76, No. 046603.

(6) Zhou, J.; Man, X.; Jiang, Y.; Doi, M. Structure formation in softmatter solutions induced by solvent evaporation. Adv. Mater. 2017, 29, No. 1703769.
(7) Schulz, M.; Keddie, J. L. A critical and quantitative review of the stratification of particles during the drying of colloidal films. Soft Matter 2018, 14, 6181-6197.

(8) Cairncross, R. A.; Francis, L. F.; Scriven, L. E. Predicting drying in coatings that react and gel: Drying regime maps. AIChE J. 1996, 42, $55-67$.

(9) Routh, A. F.; Zimmerman, W. B. Distribution of particles during solvent evaporation from films. Chem. Eng. Sci. 2004, 59, 2961-2968.

(10) Cardinal, C. M.; Jung, Y. D.; Ahn, K. H.; Francis, L. F. Drying regime maps for particulate coatings. AIChE J. 2010, 56, 2769-2780.

(11) Nikiforow, I.; Adams, J.; König, A. M.; Langhoff, A.; Pohl, K.; Turshatov, A.; Johannsmann, D. Self-stratification during film formation from latex blends driven by differences in collective diffusivity. Langmuir 2010, 26, 13162-13167.

(12) Trueman, R. E.; Lago Domingues, E.; Emmett, S. N.; Murray, M. W.; Routh, A. F. Auto-stratification in drying colloidal dispersions: A diffusive model. J. Colloid Interface Sci. 2012, 377, 207-212.

(13) Trueman, R. E.; Lago Domingues, E.; Emmett, S. N.; Murray, M. W.; Keddie, J. L.; Routh, A. F. Autostratification in drying colloidal dispersions: Experimental investigations. Langmuir 2012, 28, 34203428.

(14) Atmuri, A. K.; Bhatia, S. R.; Routh, A. F. Autostratification in drying colloidal dispersions: Effect of particle interactions. Langmuir 2012, 28, 2652-2658.

(15) Cheng, S.; Grest, G. S. Molecular dynamics simulations of evaporation-induced nanoparticle assembly. J. Chem. Phys. 2013, 138, No. 064701.

(16) Doumenc, F.; Salmon, J.-B.; Guerrier, B. Modeling flow coating of colloidal dispersions in the evaporative regime: Prediction of deposit thickness. Langmuir 2016, 32, 13657-13668.

(17) Howard, M. P.; Reinhart, W. F.; Sanyal, T.; Shell, M. S.; Nikoubashman, A.; Panagiotopoulos, A. Z. Evaporation-induced assembly of colloidal crystals. J. Chem. Phys. 2018, 149, No. 094901.

(18) Fortini, A.; Martín-Fabiani, I.; De La Haye, J. L.; Dugas, P.-Y.; Lansalot, M.; D’Agosto, F.; Bourgeat-Lami, E.; Keddie, J. L.; Sear, R. P. Dynamic stratification in drying films of colloidal mixtures. Phys. Rev. Lett. 2016, 116, No. 118301.

(19) Martín-Fabiani, I.; Fortini, A.; Lesage de la Haye, J.; Koh, M. L.; Taylor, S. E.; Bourgeat-Lami, E.; Lansalot, M.; D’Agosto, F.; Sear, R. P.; Keddie, J. L. pH-switchable stratification of colloidal coatings: surfaces "on demand". ACS Appl. Mater. Interfaces 2016, 8, 3475534761.

(20) Zhou, J.; Jiang, Y.; Doi, M. Cross interaction drives stratification in drying film of binary colloidal mixtures. Phys. Rev. Lett. 2017, 118, No. 108002.

(21) Fortini, A.; Sear, R. P. Stratification and size segregation of ternary and polydisperse colloidal suspensions during drying. Langmuir 2017, 33, 4796-4805.

(22) Howard, M. P.; Nikoubashman, A.; Panagiotopoulos, A. Z. Stratification dynamics in drying colloidal mixtures. Langmuir 2017, 33, 3685-3693.

(23) Howard, M. P.; Nikoubashman, A.; Panagiotopoulos, A. Z. Stratification in drying polymer-polymer and colloid-polymer mixtures. Langmuir 2017, 33, 11390-11398.

(24) Makepeace, D. K.; Fortini, A.; Markov, A.; Locatelli, P.; Lindsay, C.; Moorhouse, S.; Lind, R.; Sear, R. P.; Keddie, J. L. Stratification in binary colloidal polymer films: Experiment and simulations. Soft Matter 2017, 13, 6969-6980.

(25) Sear, R. P.; Warren, P. B. Diffusiophoresis in nonadsorbing polymer solutions: The Asakura-Oosawa model and stratification in drying films. Phys. Rev. E 2017, 96, No. 062602.

(26) Sear, R. P. Stratification of mixtures in evaporating liquid films occurs only for a range of volume fractions of the smaller component. J. Chem. Phys. 2018, 148, No. 134909.

(27) Liu, X.; Liu, W.; Carr, A. J.; Vazquez, D. S.; Nykypanchuk, D.; Majewski, P. W.; Routh, A. F.; Bhatia, S. R. Stratification during evaporative assembly of multicomponent nanoparticle films. J. Colloid Interface Sci. 2018, 515, 70-77. 
(28) Tatsumi, R.; Iwao, T.; Koike, O.; Yamaguchi, Y.; Tsuji, Y. Effects of the evaporation rate on the segregation in drying bimodal colloidal suspensions. Appl. Phys. Lett. 2018, 112, No. 053702.

(29) Cusola, O.; Kivistö, S.; Vierros, S.; Batys, P.; Ago, M.; Tardy, B. L.; Greca, L. G.; Roncero, M. B.; Sammalkorpi, M.; Rojas, O. J. Particulate coatings via evaporation-induced self-assembly of polydisperse colloidal lignin on solid interfaces. Langmuir 2018, 34, 5759-5771.

(30) Tang, Y.; Grest, G. S.; Cheng, S. Stratification in drying films containing bidisperse mixtures of nanoparticles. Langmuir 2018, 34, $7161-7170$.

(31) Carr, A. J.; Liu, W.; Yager, K. G.; Routh, A. F.; Bhatia, S. R. Evidence of stratification in binary colloidal films from microbeam Xray scattering: Toward optimizing the evaporative assembly processes for coatings. ACS Appl. Nano Mater. 2018, 1, 4211-4217.

(32) Statt, A.; Howard, M. P.; Panagiotopoulos, A. Z. Influence of hydrodynamic interactions on stratification in drying mixtures. $J$. Chem. Phys. 2018, 149, No. 024902.

(33) Martín-Fabiani, I.; Koh, M. L.; Dalmas, F.; Elidottir, K. L.; Hinder, S. J.; Jurewicz, I.; Lansalot, M.; Bourgeat-Lami, E.; Keddie, J. L. Design of waterborne nanoceria/polymer nanocomposite UVabsorbing coatings: Pickering versus blended particles. ACS Appl. Nano Mater. 2018, 1, 3956-3968.

(34) Routh, A. F.; Russel, W. B. Horizontal drying fronts during solvent evaporation from latex films. AIChE J. 1998, 44, 2088-2098.

(35) Everaers, R.; Ejtehadi, M. R. Interaction potentials for soft and hard ellipsoids. Phys. Rev. E 2003, 67, No. 041710.

(36) in 't Veld, P. J.; Plimpton, S. J.; Grest, G. S. Accurate and efficient methods for modeling colloidal mixtures in an explicit solvent using molecular dynamics. Comput. Phys. Commun. 2008, 179, 320329.

(37) Cheng, S.; Grest, G. S. Structure and diffusion of nanoparticle monolayers floating at liquid/vapor interfaces: A molecular dynamics study. J. Chem. Phys. 2012, 136, No. 214702.

(38) in 't Veld, P. J.; Petersen, M. K.; Grest, G. S. Shear thinning of nanoparticle suspensions. Phys. Rev. E 2009, 79, No. 021401.

(39) Plimpton, S. Fast parallel algorithms for short-range molecular dynamics. J. Comput. Phys. 1995, 117, 1-19.

(40) Piazza, R.; Parola, A. Thermophoresis in colloidal suspensions. J. Phys.: Condens. Matter 2008, 20, No. 153102.

(41) Brenner, H. Phoresis in fluids. Phys. Rev. E 2011, 84, No. 066317 .

(42) Cheng, S.; Lechman, J. B.; Plimpton, S. J.; Grest, G. S. Evaporation of Lennard-Jones fluids. J. Chem. Phys. 2011, 134, No. 224704.

(43) Tang, Y.; Grest, G. S.; Cheng, S. Stratification of drying particle suspensions: Comparison of implicit and explicit solvent simulations. arXiv:1810.05483. arXiv.org e-Print archive. https://arxiv.org/pdf/ 1810.05483 (submitted Oct 12, 2018).

(44) Beaglehole, D. Structural changes in the surface of water. J. Phys. Chem. 1987, 91, 5091-5092.

(45) Kuz, V.; Garazo, A.; Fasano, S. Surface temperature of a liquidvapor interface. J. Colloid Interface Sci. 1989, 133, 511-513.

(46) Price, P. E.; Cairncross, R. A. Optimization of single-zone drying of polymer solution coatings using mathematical modeling. $J$. Appl. Polym. Sci. 2000, 78, 149-165.

(47) Krantz, W. B.; Greenberg, A. R.; Hellman, D. J. Dry-casting: Computer simulation, sensitivity analysis, experimental and phenomenological model studies. J. Membr. Sci. 2010, 354, 178-188.

(48) Dünweg, B. Molecular dynamics algorithms and hydrodynamic screening. J. Chem. Phys. 1993, 99, 6977-6982.

(49) Tsige, M.; Grest, G. S. Molecular dynamics simulation of solvent-polymer interdiffusion: Fickian diffusion. J. Chem. Phys. 2004, 120, 2989-2995. 